\title{
Integration of Database Management System with Cloud
}

\author{
Sushma V \\ GSSSIETW, \\ Mysuru, India
}

\begin{abstract}
In recent years, outsourcing the database has become an important component of cloud computing. Due to the continuous advancements in a network technology, the cost required to transmit a terabyte of data over long distances as decreased significantly in the past decade. In addition, the total cost of data management is five to ten times higher than the initial acquisition cost. As a result, there is a growing interest in outsourcing database management tasks to third parties that can provide these tasks for much lower cost due to the economy of scale. This new outsourcing model has the benefits of reducing the cost for running Database Management System (DBMS) independently.
\end{abstract}

Keywords: SQL (Structured Query Language), RDBMS (Relational Database Management System), BigData, IaaS(Infrastructure as a service), PaaS(Platform as a service component)

\section{INTRODUCTION}

A Cloud database management system is a distributed database that delivers computing as a service instead of a product. It is the sharing of resources, software, and information between multiple devices over a network which is mostly the internet. It is expected that this number will grow significantly in the future. As a result, there is a growing interest in outsourcing database management tasks to third parties that can provide these tasks for much lower cost due to the economy of scale just like putting it into the cloud. In this paper, we discuss the recent trend in database management system and the possibilities of making it as one of the services offered in the cloud.

\section{THE TRADITIONAL WAY}

Storing of data on computer hard disk and secondary storage is too main stream and has been facing many problems these days, due to data loss, failure and difficulty in remote access by concurrent users. To avoid data loss and allow concurrent users access, Cloud Computing has been put into use which allows data to be stored on a virtual cloud like storage area, which can be accessed by a user from remote location and data is even safe. Using Database as a Cloud Service allows the user to access the data stored on an online database (present on a cloud) and is hence proving to be an efficient technological advancement these days.

\section{DBMS AS A CLOUD SERVICE}

Most DBMS or database management systems are simply software packages that users can acquire to create, maintain or use a database. However, since the introduction of cloud computing, DBMS has morphed into an entirely new type of service with its own unique benefits and task specific advantages. For one thing, any type of cloud service model will have to employ a dedicated cloud DBMS in order to truly provide customers with excellent access to data and databases. Traditional DBMS's are simply not set up or equipped to deal with the demands of cloud computing. And of course, if DBMS was deployed as a service as part of a larger package provided, it would likely be much more efficient in its duties and therefore cheaper in the long run.

The concept of the DBMS has been around since the beginning of commercial computing; such as the navigational DBMS of the1960's. Database management systems are one of the oldest integral components of computing, essentially making it possible to scan, retrieve and organize data on hard drives and networks. All DBMS, despite whether traditional or cloud-based, are essentially communicators that function as middlemen between the operating system and the database.

How is a cloud DBMS different a traditional one? For one thing, cloud-based DBMS are extremely scalable. They are able to handle volumes of data and processes that would exhaust a typical DBMS. Despite their scalability however, cloud DBMS are still somewhat lacking in their ability to scale up to extremely large processes; this is expected to be remedied in the coming months and years however. Currently, the use of cloud DBMS's are principally used in the testing and development of new cloud applications and processes[1]. But while a stand-alone DBMS can be used on a cloud infrastructure; most are not designed to take full advantage of cloud resources. DBMS as a cloud service-type models seek to capitalize on the disparity between antiquated DBMS models and their lack of full cloud functionality.

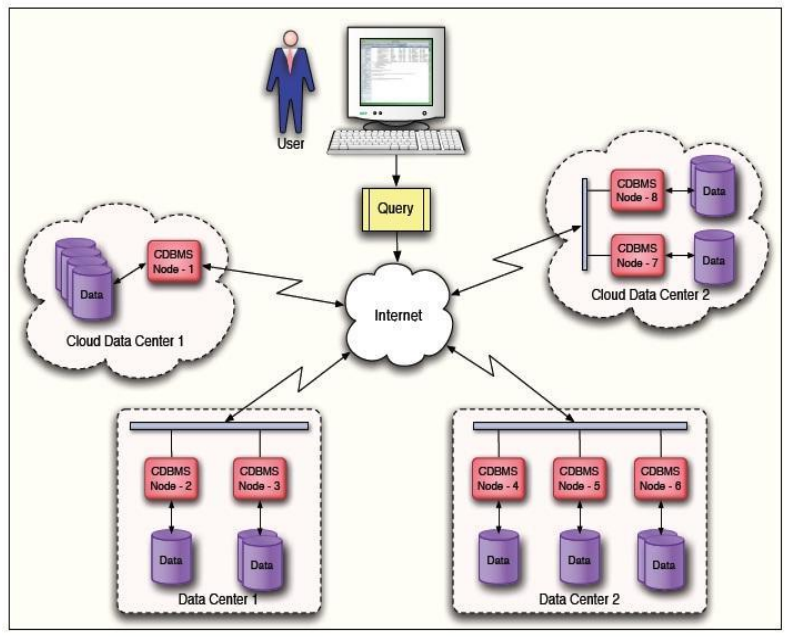

Figure. 1 Basic model of Cloud Database

\section{CLOUD DATABASE STRATEGY}

One of the most significant (and complex) cloud infrastructure issues facing cloud providers of many types is deciding how database support will be offered in the cloud, which is also leading to selling Database as a Service[2]. The wrong cloud 
database strategy can create application performance problems significant enough to discredit a cloud service, forcing the provider to incur additional costs to establish credibility with users.

The cloud database issue is complicated because it sits at the intersection of two cloud infrastructure models, two storage service models and two database management systems (DBMS) models. Sorting out the details will require cloud to consider their infrastructure, network performance and service goals.

The following services models can affect cloud database support:

\subsection{Single and multi-site cloud infrastructure models}

The two cloud infrastructure models differ in the way that resources are allocated to customers. In the single-site model, a customer's applications run within a single data centre in the cloud, even if multiple data centres are available. This means that the storage and/or DBMS resources used by a customer can be contained within a single storage area network (SAN), and that the customer's application performance in the cloud can likely match that of a standard data centre that uses virtualization. In the multi-site model, the customer's applications can draw on resources from multiple data centres, which mean that making the connection between the application and the database resources could involve WAN connectivity that limits performance[3]. Whichever choice they make, service providers must be ready to address the issues that come with single- or multi-site cloud infrastructure.

\subsection{Storage and database service models}

The storage service models available to a cloud planner are Storage as a Service or the more complex Database as a Service. With storage services, the customer will access virtual storage devices as though they were native disk arrays, which means that the applications will send storage protocols (such as Fibre Channel over Ethernet or IP SCSI) over any network connection. In the relatively new Database as Service offerings, applications will access storage through a cloud DBMS that will accept high-level database commands and return the required results. This can create a less delaysensitive connection, so it is better suited to cloud configurations where storage might be distributed over multiple sites.

Another major cloud database planning decision is whether a cloud database service should be based on the popular relational database management system (RDBMS) and its Structured Query Language (SQL) standards, based on a lighter-weight RDBMS without SQL, or based on a nonrelational structure like the 'Google BigTable' structure that gives users dynamic control over data layout and format

\section{DBMS IN CLOUD ARCHITECTURE}

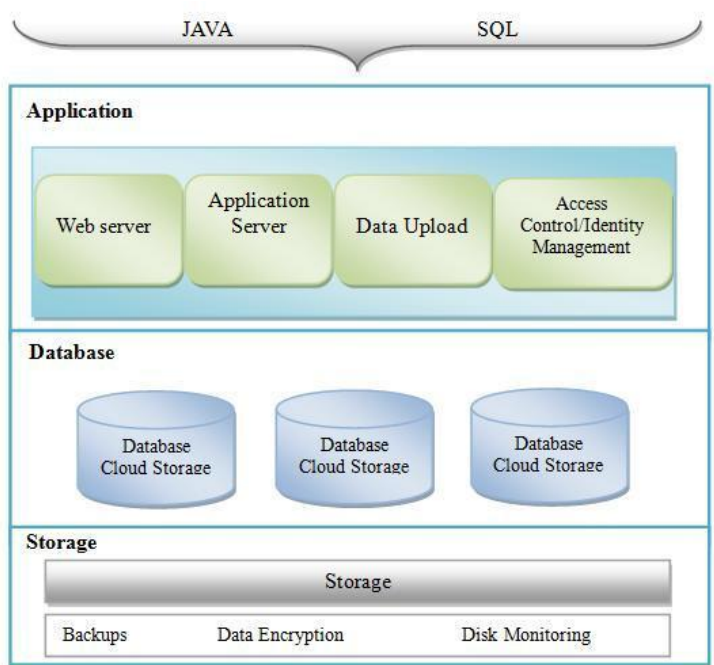

Figure 2 DBMS in Cloud Architecture

Above is a proposed DBMS in Cloud Architecture, first layer is the storage, followed by databases and the upper layer is application layer. In terms of performance, it provides efficient data access with a better distribution of values for some data. Stores frequently used SQL statements in memory, avoiding the need for time-consuming recompilation at runtime[4]. Produces a detailed report on each step used for data access, allowing you to accurately implement performance enhancements. Data is encrypted when stored or backed up, without any need for programming to encrypt and decrypt

\section{THE VALUE OF DBMS AS A CLOUD SERVICE}

\subsection{Advantages}

1) Database as a Service has advantages beyond marketing. With a cloud DBMS, storage virtualization isn't directly visible to the applications, which gives operators more latitude in how they manage storage resources.

2) With a direct storage model, a mechanism for storage virtualization that protects customers' data from being accessed by others but still makes the virtual disks look "real" is essential

3) It helps in controlling the performance of applications that use storage extensively

4) It's easy to provide cloud database services as part of a cloud Platform as a Service (PaaS) offering, but the

5) applications may have to be written to access cloud database services in some Infrastructure as a Service (IaaS) configurations[5].

6) 5) Offering Database as a Service can help by replacing storage input/output (I/O) with simply sending a query and a return of results. 


\subsection{Demerits}

1) If customers are likely to access the storage/DBMS across multiple data centres in cloud, the performance implications may be critical.

2) Privacy has been the most important issue when it comes to cloud computing. The companies cannot afford to leak out information that is stored in their database. If there is encryption of data in the database, then it is quite easy to store it in an easy way.

3) Query and Transactional workload: Transactional workload determines the overall time that will be required[6]. But we cannot determine the Query workload, since there can be multiple users firing numerous queries at same time, which would be time consuming.

4) Internet Speed: The speed of data transfer in the data centre is comparatively very high as compared to the speed of internet that is used to access the data centre. This is a barrier to the performance of cloud database[7].

\section{CONCLUSION}

Database Management Systems as a cloud service are engineered to run as a scalable, elastic service available on a cloud infrastructure. Cloud DBMSs will have an impact for vendors desiring a less expensive platform for development. In this paper, we presented the idea of DBMS in the cloud, the possibilities to be offered as one of the services offered by promising capability of cloud computing, that is to be a DBMS as a Service. In this paper we proposed architecture of DBMS in the cloud.

\section{REFERENCES}

[1] A. Thusoo, J. S. Sarma, N. Jain, Z. Shao, P. Chakka, S. Anthony, H. Liu, P. Wyckoff, and R. Murthy, "Hive: a warehousing solution over a map-reduce framework," Proc. VLDB Endow., vol. 2, pp. 1626-1629, August 2009.

[2] M. Stonebraker, S. Madden, D. J. Abadi, S. Harizopoulos, N. Hachem, and P. Helland. The end of an architectural era: (it's time for a complete rewrite). In VLDB, pages 1150-1160, 2007

[3] J. Lee, M. Muehle, N. May, F. Faerber, V. S. H. Plattner, J. Krueger, and M. Grund. High-performance transaction processing in SAP HANA. IEEE Data Eng. Bull., 36(2):28-33, 2013.

[4] GravelleR, "Should You Move Your MySQL Database to the Cloud?", http://www.databasejournal.com/ features/mssql/should-you-move-your-mysql-database-tothecloud.html.

[5] A. Williamson, "Comparing cloud computing providers," Cloud Comp. J., vol. 2, no. 3, pp. 3-5, 2009.

[6] B. R. Kandukuri, R. Paturi V, A. Rakshit, "Cloud Security Issues", In Proceedings of IEEE International Conference on Services Computing, pp. 517-520, 2009.

[7] V. Krishna Reddy, B. Thirumal Rao, Dr. L.S.S. Reddy, P.Sai Kiran "Research Issues in Cloud Computing " Global Journal of Computer Science and Technology, Volume 11, Issue 11, July 2011. 